\title{
Copolymerization of 4-Acetylphenyl Methacrylate with Ethyl Methacrylate: Synthesis, Characterization, Monomer Reactivity Ratios, and Thermal Properties
}

\author{
Gamze Barim ${ }^{1}$ and Mustafa Gokhun Yayla ${ }^{2}$ \\ ${ }^{1}$ Department of Chemistry, Faculty of Science and Arts, University of Adiyaman, 02040 Adiyaman, Turkey \\ ${ }^{2}$ Physics Engineering Department, Faculty of Science and Letters, Istanbul Technical University, Maslak, 34469 Istanbul, Turkey \\ Correspondence should be addressed to Gamze Barim; gbarim@gmail.com
}

Received 27 August 2013; Revised 2 November 2013; Accepted 3 November 2013; Published 4 February 2014

Academic Editor: Giridhar Madras

Copyright ( $) 2014$ G. Barim and M. G. Yayla. This is an open access article distributed under the Creative Commons Attribution License, which permits unrestricted use, distribution, and reproduction in any medium, provided the original work is properly cited.

\begin{abstract}
Methacrylates have high glass transition temperature $\left(T_{g}\right)$ values and high thermal stability. A new methacrylate copolymer, poly(4acetylphenyl methacrylate-co-ethyl methacrylate) (APMA-co-EMA), was synthesized. The thermal behaviors of copolymers were investigated by differential scanning calorimetry and thermogravimetric analysis. They behaved as new single polymers with single $T_{g}$ 's and the thermal stability of the copolymers increased with increasing 4-acetylphenyl methacrylate (APMA) fraction, leading to the manufacture of copolymers with desired $T_{g}$ values. Structure and composition of copolymers for a wide range of monomer feed ratios were determined by Fourier transform infrared (FT-IR) and ${ }^{1} \mathrm{H}$-nuclear magnetic resonance $\left({ }^{1} \mathrm{H}-\mathrm{NMR}\right)$ spectroscopic techniques. Copolymerization reactions were continued up to $40 \%$ conversions. The monomer reactivity ratios for copolymer system were determined by the Kelen-Tüdös $\left(r_{a(\mathrm{APMA})}=0.81 ; r_{b \text { (EMA })}=0.61\right)$ and extended Kelen-Tüdös $\left(r_{a}=0.77 ; r_{b}=0.54\right)$ methods and a nonlinear least squares $\left(r_{a}=0.74 ; r_{b}=0.49\right)$ method.
\end{abstract}

\section{Introduction}

In recent years, many studies have been carried on aromatic acrylate and methacrylate monomers and polymers [1-4]. They are highly reactive monomers because of the presence of the aromatic ring and thus form an interesting class of polymers [5-7]. Poly(phenyl methacrylates) have higher thermal stability and gain much interest due to their potential industrial uses [8-10]. Copolymers of phenyl methacrylate with various acrylates are reported as adhesives for leather to cloth bonding [11-15]. Poly(phenyl methacrylate)s are harder polymers of higher tensile strength and find applications in pressure-sensitive, photo imaging materials [16] and in electrophotographic photoreceptors for offset printing plates [17]. Other uses include photo luminescent [18], photo resistive [19], photosensitive materials [20], biomaterials [21, 22], optical telecommunication materials [23], and a polymer supported catalyst [24].
The chemical composition of the copolymers and distribution of the monomer units on the chain depend on their incorporation rates which in turn depend on their reactivity ratios. Monomer reactivity ratios are essential quantitative values to predict the copolymer composition for any starting feed ratio and to understand the kinetic and mechanistic aspects of copolymerization [25-27].

To the best of the author's knowledge there are no reports in the literature on APMA with ethyl methacrylate (EMA) copolymerization. Reddy et al. have investigated the copolymerization of another phenyl monomer ( $\mathrm{n}$-phenyl methacrylamide) with EMA [28]. Roy et al. have investigated the RAFT copolymerization of $\mathrm{N}, \mathrm{N}$-(dimethylamino)ethyl methacrylate and methyl methacrylate [29]. There are also studies related to the synthesis and reactivity ratios of copolymers of APMA with glycidyl methacrylate [30]. All these studies were low conversion investigations. On the other hand the industry always works at moderate or high conversion. There 
<smiles>C=C(C)C(=O)Oc1ccc(C(C)=O)cc1</smiles><smiles>C=C(C)C(=O)Oc1ccc(C(C)=O)cc1</smiles>

Scheme 1: Synthesis of APMA and poly(APMA-co-EMA).

are no moderate or high conversion investigations related to determining reactivity ratios of APMA-co-EMA system.

For this reason, in this study the synthesis and characterization of copolymers of APMA with EMA are investigated at moderate conversion. The monomer reactivity ratios are calculated using three different methods: Kelen-Tüdös (KT) [31], extended Kelen-Tüdös (EKT) [32], and a nonlinear least squares (NLLS) [33]. The EKT and the NLLS methods take the composition drift into account and are suitable for high conversion experiments.

$T_{g}$ 's of new copolymers and the effect of APMA content on the thermal properties of resulting copolymers are also investigated.

\section{Experimental}

2.1. Materials. 4-Hydroxy acetophenone (Aldrich) and methacryloyl chloride (Merck) were used as received. EMA was freed from inhibitor by being washed with $5 \%$ sodium hydroxide solution several times, followed by being washed with distilled water, and then was dried over anhydrous sodium sulphate; subsequently it was distilled under reduced pressure. $2-2^{\prime}$-Azobisisobutyronitrile (AIBN) was recrystallized from chloroform and methanol mixture. 1,4-Dioxane, triethylamine, tetrahydrofuran (THF), sodium hydroxide, sodium sulphate (Merck), and ethanol (Merck) were of analytical grade and were used as received.

2.2. Synthesis of 4-Acetylphenyl Methacrylate. APMA monomer was prepared from reaction of 4-hydroxy acetophenone with methacryloyl chloride in the presence of triethylamine and THF at 0 to $5^{\circ} \mathrm{C}$ range [26] (Scheme 1, the first row).
The monomer was characterized by FT-IR and ${ }^{1} \mathrm{H}-\mathrm{NMR}$ spectra as follows:

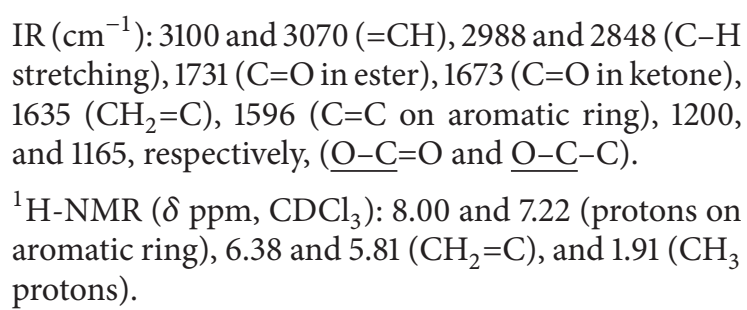

2.3. Homopolymerization and Copolymerization. Homopolymerization and copolymerization reactions were carried out in 1,4 -dioxane solution at $70^{\circ} \mathrm{C}$ using AIBN ( $1 \%$ based on the total weight of the monomers) as the initiator (Scheme 1, the second row). Required amounts of APMA and EMA with 1,4-dioxane and AIBN were mixed in a polymerization tube, purged with $\mathrm{N}_{2}$ atmosphere for $15 \mathrm{~min}$. Polymerization was performed at $70^{\circ} \mathrm{C}$ in a thermostatic oil bath. After 3 hours, the copolymers were precipitated in excess ethanol and filtered. The precipitated polymers were purified by repeated reprecipitation from chloroform solution using ethanol and finally dried in a vacuum oven at $40^{\circ} \mathrm{C}$ for 24 hours. The conversion was determined by gravimetric measurements. The reaction scheme of the copolymer is shown in Scheme 1. Initial monomer compositions and copolymer conversions are given in the second, third, and fourth columns and final monomer compositions are given in the fifth and sixth columns of Table 1.

2.4. Polymer Characterization. Infrared spectra were obtained with a Perkin Elmer Spectrum One FT-IR system and recorded using universal ATR sampling accessory within 


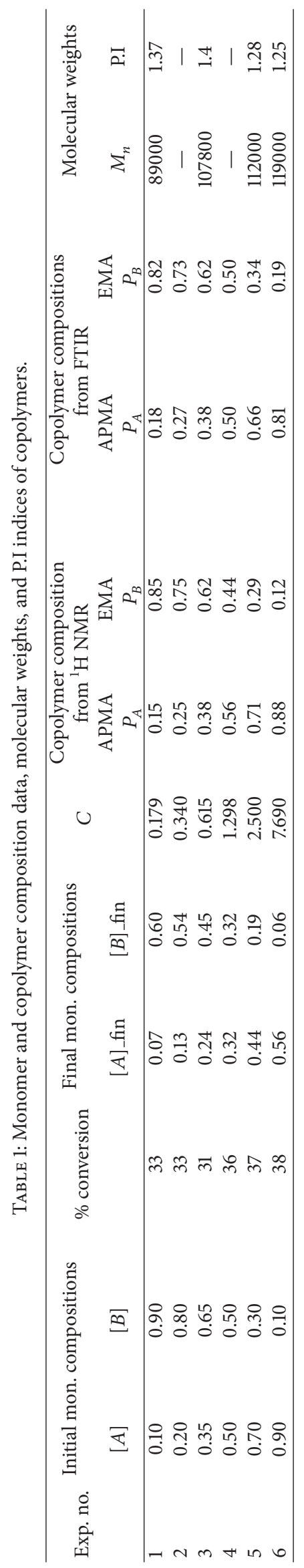


the wavelengths of $4000-650 \mathrm{~cm}^{-1}$. ${ }^{1} \mathrm{H}-\mathrm{NMR}$ spectra of copolymers were recorded in deuterated chloroform $\left(\mathrm{CDCl}_{3}\right)$ solution on a $400 \mathrm{MHz}$ Bruker AVIII 400 using tetramethylsilane as an internal reference.

The molecular weights and polydispersity index (P.I) of all polymers were determined by using gel permeation chromatography (GPC). The GPC consisted of an Agilent Iso Pump, a refractive index detector, and both Mixed " $D$ " and Mixed "E" columns (ex. Polymer Labs). THF was used as the eluent, and poly(methyl metacrylate)s were employed as standards for calibration.

The glass transition temperatures were measured by a Shimadzu DSC 50 thermal analyzer under nitrogen atmosphere at a heating rate of $10^{\circ} \mathrm{C} / \mathrm{min}$. Thermal stabilities of the polymers were investigated with a Perkin Elmer TGA/DTA 7300 .

\section{Results and Discussion}

${ }^{1} \mathrm{H}-\mathrm{NMR}$ spectra of homo- and copolymers are given in Figure 1. The chemical shifts assignments for the copolymers were based on the chemical shifts observed for the respective homopolymers. In the copolymers, the resonance signals of aromatic protons were shown between 8.00 and $7.28 \mathrm{ppm}$. And the peak at $4.06 \mathrm{ppm}$ is due to the $\mathrm{OCH}_{2}$ protons of EMA unit. Copolymer compositions were calculated from peak integrations of ${ }^{1} \mathrm{H}-\mathrm{NMR}$ signals. Thus, the mole fraction of APMA in copolymer was determined by measuring the integrated peak areas of the aromatic protons of APMA at $8.00 \mathrm{ppm}$, which is adjacent to the carbonyl and $\mathrm{OCH}_{2}$ protons of EMA at $4.06 \mathrm{ppm}$. The following equation was used to determine the composition of copolymers.

Let $P_{A}$ be the mole fraction of APMA unit and $P_{B}=(1-$ $\left.P_{A}\right)$ that of the EMA unit:

$$
\begin{aligned}
& \frac{2 P_{A}}{2\left(1-P_{A}\right)} \\
& =\frac{\text { Integral intensities of aromatic protons adjacent to the carbonyl }}{\text { Integral intensities of } \mathrm{OCH}_{2} \text { protons }} \\
& =\mathrm{C} .
\end{aligned}
$$

On simplification,

$$
P_{A}=\frac{C}{C+1} .
$$

The value of $C$ corresponding mole fractions of APMA in the copolymer is given in the seventh column of Table 1 .

FT-IR spectra of homo- and copolymers are given in Figure 2. In the copolymers, the peaks at 1751 and $1724 \mathrm{~cm}^{-1}$ were attributed to the ester carbonyl stretching of APMA and EMA units, respectively. Besides, the ketone carbonyl stretching shows absorption band at $1685 \mathrm{~cm}^{-1}$ APMA units. In the IR spectrum, the ester carbonyl stretching vibration in APMA shifted from $1731 \mathrm{~cm}^{-1}$ to $1751 \mathrm{~cm}^{-1}$ due to removal of conjugation with polymerization. Copolymer compositions were also determined from IR technique. The absorption ratios between characteristic analytical bands of
$1685 \mathrm{~cm}^{-1}$ (for APMA unit) and $1724 \mathrm{~cm}^{-1}$ (for EMA unit) $\left(A=\log \left(I_{o} / I\right)\right)$ and the least changing absorption band of $1357 \mathrm{~cm}^{-1}$ as a standard band were used to determine the copolymer compositions. Copolymer compositions were calculated from FT-IR using

$$
\begin{aligned}
& P_{A}=\frac{\Delta A^{1685} / M_{\mathrm{APMA}}}{\Delta A^{1685} / M_{\mathrm{APMA}}+\Delta A^{1724} / M_{\mathrm{EMA}}} 100, \\
& P_{B}=\frac{\Delta A^{1724} / M_{\mathrm{EMA}}}{\Delta A^{1685} / M_{\mathrm{APMA}}+\Delta A^{1724} / M_{\mathrm{EMA}}} 100,
\end{aligned}
$$

where $\Delta A=A^{i} / A^{1357}$ (least changing standard band) and $M_{\mathrm{APMA}}$ and $M_{\mathrm{EMA}}$ are molecular weights of APMA and EMA monomer units, respectively.

Copolymer compositions determined by NMR technique [27] are given in the eighth and ninth columns of Table 1 and IR technique [34] is given in the tenth and eleventh columns of Table 1. The slight discrepancies between the solution NMR and solid state FTIR measurements can be attributed to inhomogeneity of the solid sample and other measurement errors.

The number average molecular weights (Mn) and polydispersity index (P.I) of the polymer samples are given in the last two columns of Table 1 . The molecular weights increased with increasing percentage of APMA in the feed composition. The molecular weights of the monomers are $204 \mathrm{~g} / \mathrm{mol}$ (APMA) and $114 \mathrm{~g} / \mathrm{mol}$ (EMA); APMA is about $80 \%$ heavier. The higher molecular weights of the polymers with higher APMA content are due to this difference. The polydispersity index of the copolymers ranged from 1.25 to 1.4. The value of polydispersity index means that the chain termination of polymers takes place predominantly by coupling rather than by disproportionation [35].

3.1. Monomer Reactivity Ratios. The ratio of the rates of entry of the comonomers is given by the Mayo-Lewis copolymerization equation [36]:

$$
\frac{d[A]}{d[B]}=\frac{[A]}{[B]}\left\{\frac{\left(r_{a}[A]+[B]\right)}{\left([A]+r_{b}[B]\right)}\right\} .
$$

Here, $[A]$ that represents APMA and $[B]$ that represents EMA are the molar concentrations of the two comonomers in the feed and $d[A] / d[B]$ is the molar ratio of the two monomers entering the copolymer. The parameters $r_{a}$ that represents the reactivity ratio of APMA and $r_{b}$ that represents EMA are known as the monomer reactivity ratios. The composition of the copolymer depends not only on the feed composition but also on these two parameters. For this reason, the knowledge of these ratios is essential for the manufacture of copolymers of desired properties.

Designating the initial monomer ratio by $X=[A] /[B]$ and resulting copolymer ratio by $Y=d[A] / d[B]$, further arranging $X$ and $Y$ in the form of $G=X(Y-1) / Y, F=$ $X^{2} / Y$, and $\alpha=\sqrt{F_{\min } \cdot F_{\max }}$, and using $\eta=G /(\alpha+F)$ and $\xi=F /(\alpha+F)$, Mayo-Lewis equation is transformed into the linearized Kelen-Tüdös version:

$$
\eta=\left(r_{a}+\frac{r_{b}}{\alpha}\right) \xi-\frac{r_{b}}{\alpha} \text {. }
$$




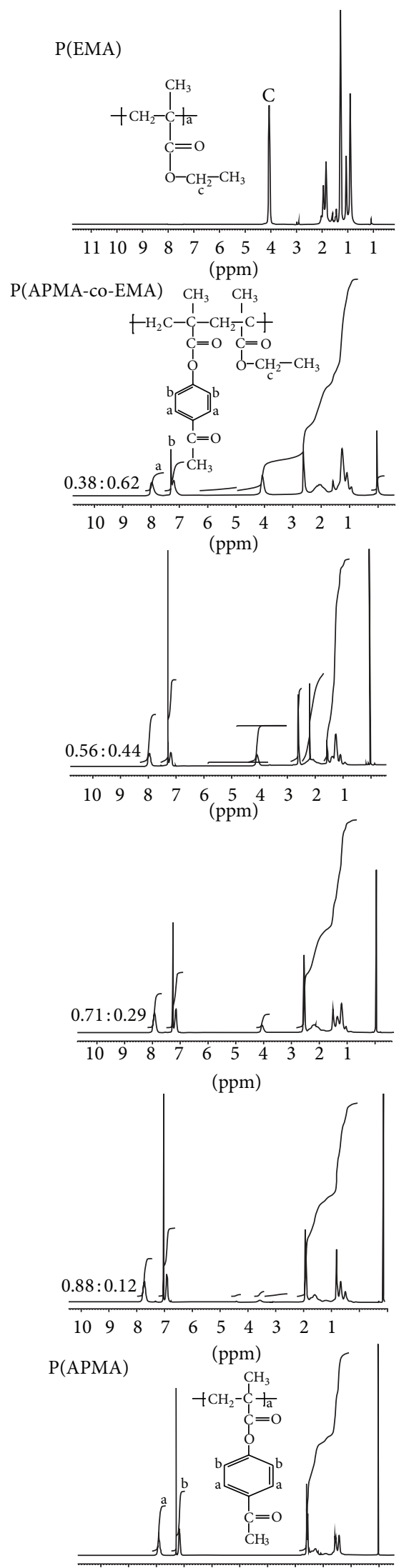

$\begin{array}{llllllllll}10 & 9 & 8 & 7 & 6 & 5 & 4 & 3 & 2 & 1\end{array}$ (ppm)

FIGURE $1:{ }^{1}$ H-NMR spectra of homopolymer and copolymers. 


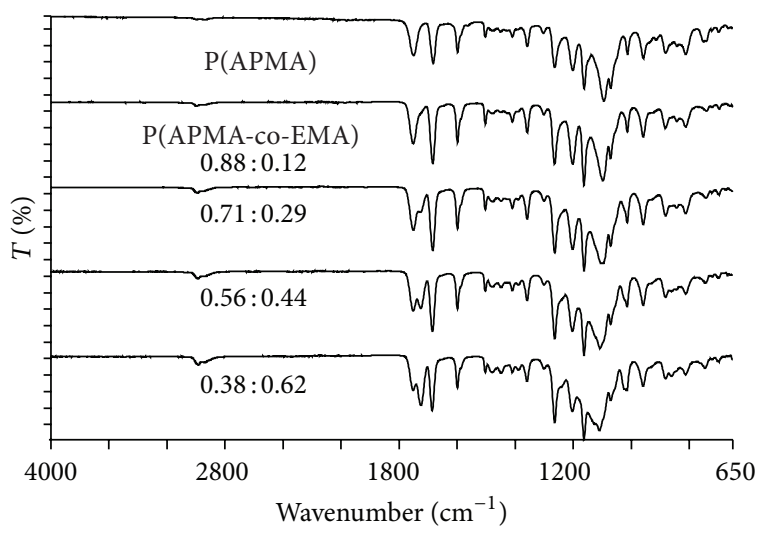

FIGURE 2: IR spectra of homopolymer and copolymers.

TABLE 2: KT parameters for APMA-co-EMA copolymer system.

\begin{tabular}{lcccccc}
\hline Polymer & $X$ & $Y$ & $G$ & $F$ & $\eta$ & $\xi$ \\
\hline 1 & 0.11 & 0.17 & -0.518 & 0.069 & -0.586 & 0.073 \\
2 & 0.25 & 0.33 & -0.500 & 0.187 & -0.432 & 0.175 \\
3 & 0.53 & 0.58 & -0.382 & 0.495 & -0.152 & 0.360 \\
4 & 1.00 & 1.27 & 0.214 & 0.785 & 0.015 & 0.472 \\
5 & 2.33 & 2.44 & 1.380 & 2.223 & 0.385 & 0.716 \\
6 & 9.00 & 7.33 & 7.772 & 11.050 & 0.702 & 0.926 \\
\hline
\end{tabular}

$\alpha=0.87905$.

TABLE 3: Extended KT parameters for APMA-co-EMA copolymer system.

\begin{tabular}{lccccccc}
\hline Polymer & $\xi_{1}$ & $\xi_{2}$ & $Z$ & $F$ & $G$ & $\eta$ & $\xi$ \\
\hline 1 & 0.493 & 0.310 & 1.828 & 0.05 & -0.45 & -0.572 & 0.060 \\
2 & 0.411 & 0.308 & 1.436 & 0.16 & -0.46 & -0.423 & 0.165 \\
3 & 0.334 & 0.308 & 1.105 & 0.47 & -0.37 & -0.132 & 0.369 \\
4 & 0.403 & 0.317 & 1.354 & 0.69 & 0.20 & -0.004 & 0.459 \\
5 & 0.375 & 0.357 & 1.062 & 2.16 & 1.36 & 0.376 & 0.726 \\
6 & 0.371 & 0.456 & 0.763 & 12.50 & 8.30 & 0.680 & 0.939 \\
\hline
\end{tabular}

$\alpha=0.81562$.

The parameters of $\mathrm{KT}$ and EKT equations are presented in Tables 2 and 3. The KT plot is given as Figure 3. The KT coefficient $\alpha=0.87$ indicates that the experimental planning was good. The data lie on the straight line with very little scatter. The monomer reactivity ratios are found as $r_{a}=0.81$ and $r_{b}=0.61$. The KT method has the advantages that the data are uniformly distributed and the results do not depend on which monomer is labeled monomer $(A)$ and which is labeled monomer $(B)$. Its disadvantage is that it uses the initial ratio of the two monomers rather than the reaction weighted cumulative average. When one monomer enters the reaction faster and is depleted more rapidly than the other, the feed composition continuously drifts during the reaction. The KT method fails to take into account this drift. Kelen and Tüdös later proposed an extended version of their method which takes the composition drift into account [32]. Since the experiments are stopped at $30-40 \%$ conversion, the composition drift can affect the results. For this reason the

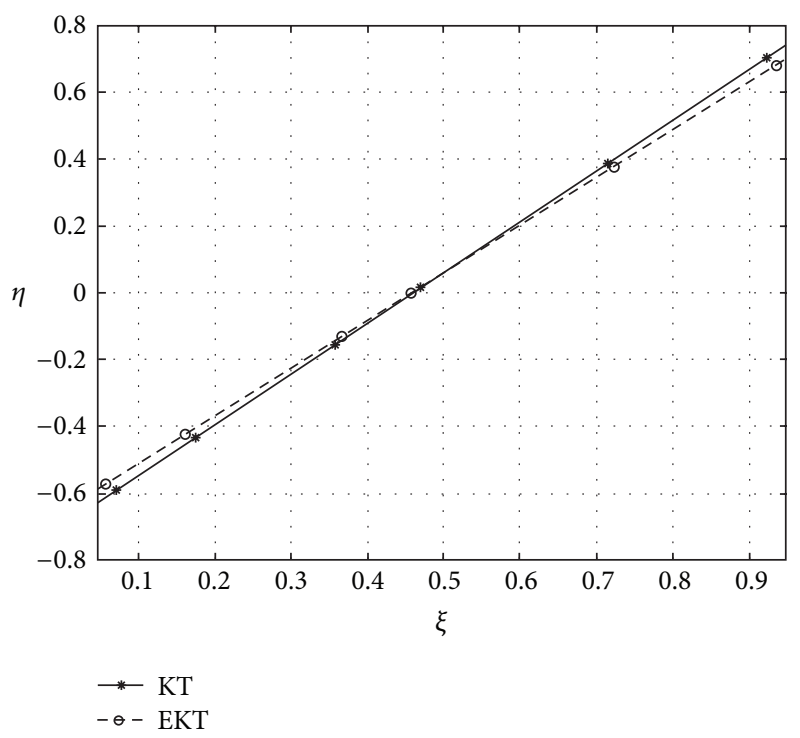

Figure 3: KT and EKT plot for APMA-EMA copolymerization.

data were reanalyzed using the extended Kelen-Tüdös (EKT) method.

EKT equation is the same as $\mathrm{KT}$ equation shown in (5). The difference between KT and EKT methods is the calculation of $F$ and $G$. In EKT method, they are calculated through $F=Y / Z^{2}, G=(Y-1) / Z$, where $Z=\log (1-$ $\left.\xi_{1}\right) / \log \left(1-\xi_{2}\right)$, and $\xi_{1}=[A]_{0} / d[A], \xi_{2}=[B]_{0} / d[B]$. Here $[A]_{0}$ and $[B]_{0}$ are the initial molar monomer fractions.

The EKT plot is given in Figure 3. The reactivity ratios are found as $r_{a}=0.77$ and $r_{b}=0.54$.

In the NLLS method used, the data is fitted to a numerical solution of the Mayo-Lewis copolymerization equation. Thus errors emanating from linearization are eliminated. For each $r_{a}, r_{b}$ pair, the copolymerization equation (4) is integrated numerically from initial monomer concentrations to final concentration of monomer $(B)$. The squares of the differences of the final calculated value of the monomer $(A)$ from its experimental value

$$
\Delta[A]=[A]_{\text {final_experimental }}-[A]_{\text {final_calculated }}
$$

are added for each experiment and the chi-square for that set of monomer reactivity ratios are calculated:

$$
\chi^{2}=\sum_{i=1}^{n} \frac{\Delta[A]_{i}^{2}}{\delta A^{2}} .
$$

Here $\delta A$ is the error bar for the relevant point. The procedure is than repeated for each pair of $r_{a}$ and $r_{b}$ in the scanning range. The map thus produced is used for the best parameter estimate and the confidence intervals. As the data is fitted to the nonlinear Mayo equation itself and not to a linearized form of it, distortion of the error structure is avoided. The resulting contour map is given as Figure 4 and monomer reactivity ratios are found as $r_{a}=0.74$ and $r_{b}=0.49$.

Using three separate methods to evaluate the reactivity ratios ensures that no algorithmic errors are present. In addition the differences of monomer reactivity ratios calculated 


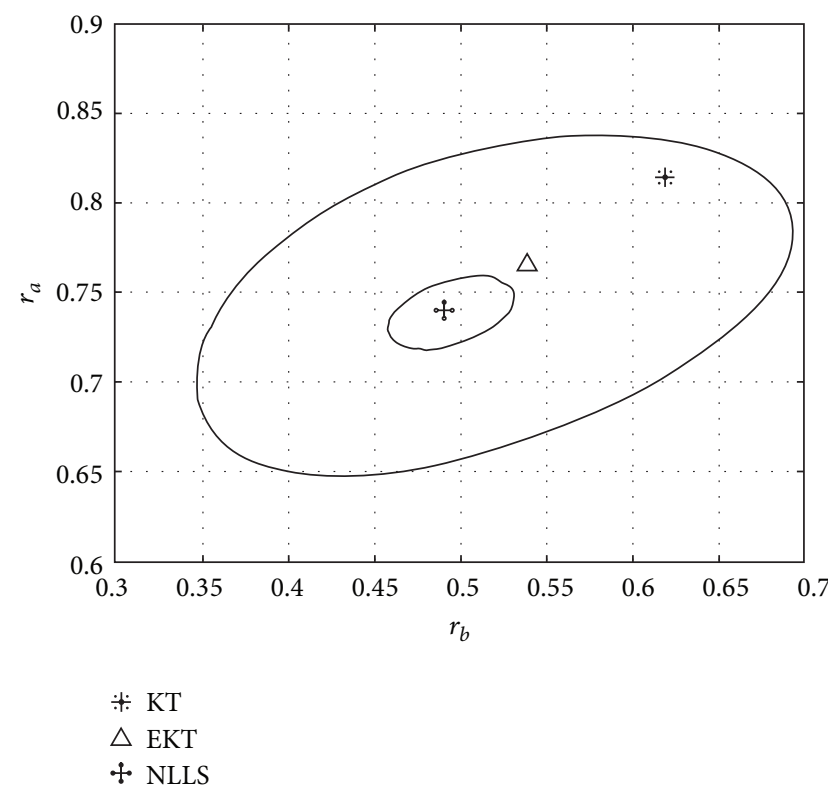

Figure 4: Probability contours and the best fit point (+) obtained by NLLS. Results of EKT $(\Delta)$ and $\mathrm{KT}(*)$ are also shown on the graph.

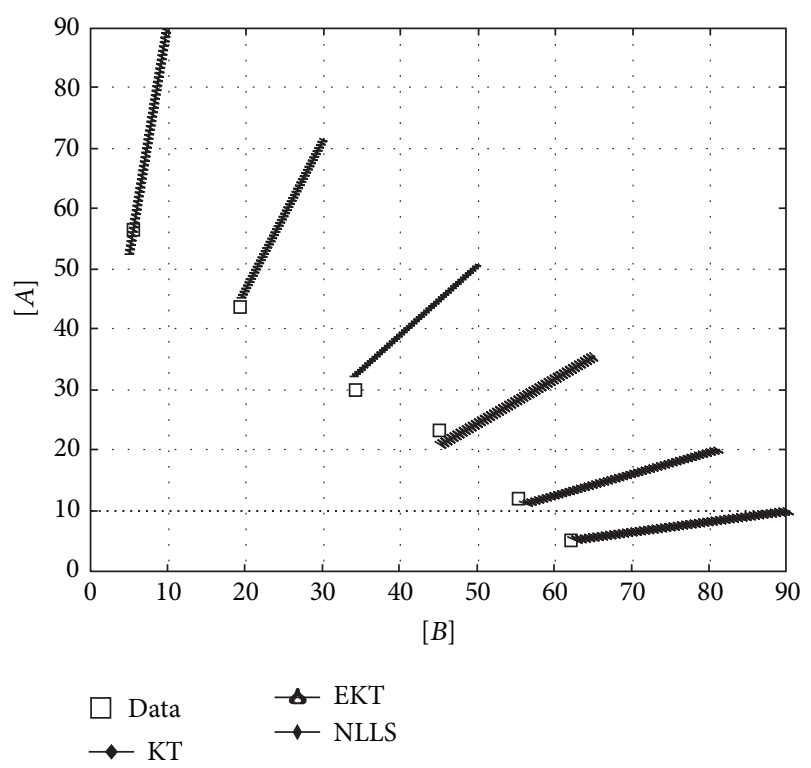

FIGURE 5: Evolution of the concentrations of the comonomers for all experiments. The molar concentrations are given as percentages of the initial total molar concentration.

by the three methods give an estimate on the dependence of the results on the method of evaluation and the weighting of data points by the three methods. The EKT and NLLS results are close to each other while the KT method gives slightly larger values (closer to one). Since the original KT method does not take composition drift into account and as the conversions ranged up to $40 \%$, this is expected.

Figure 5 shows the numerical integration of the copolymerization equation beginning from the initial conditions for each experiment using the monomer reactivity ratios given by
TABLE 4: The reactivity ratios calculated by the three methods.

\begin{tabular}{lccc}
\hline Monomer reactivity ratio & KT & EKT & NLLS \\
\hline$r_{a}$ & 0.81 & 0.77 & 0.74 \\
$r_{b}$ & 0.61 & 0.54 & 0.49 \\
\hline
\end{tabular}

$a$ : APMA; $b$ : EMA.

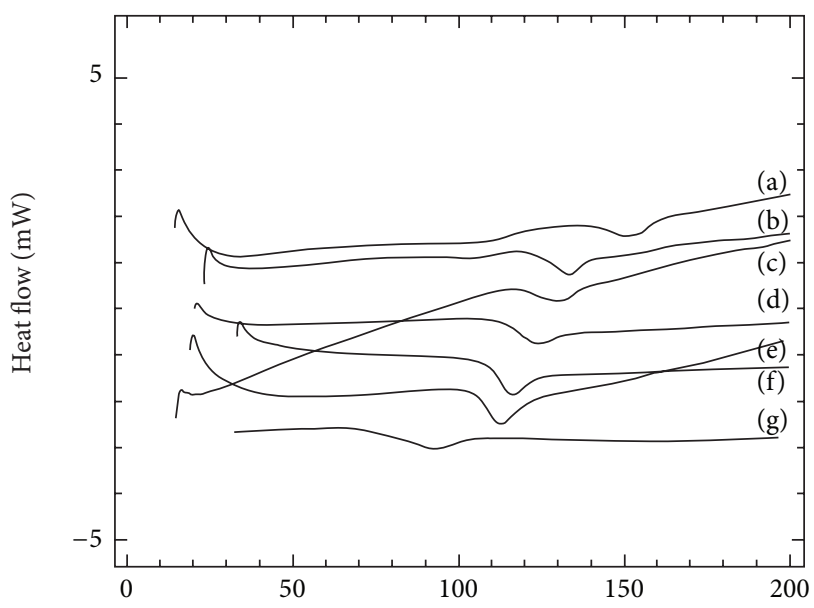

FIGURE 6: DSC thermograms of investigated copolymers; (a) poly(APMA), APMA unit in copolymer; (b) 0.88 , (c) 0.56 , (d) 0.38 , (e) 0.25 , (f) 0.15 , (g) poly(EMA).

the three calculation techniques. The three lines starting from each initial point are the numerical integration results using the reactivity ratios calculated by the three methods and the squares are the experimental data. As the monomer reactivity ratios found by the three methods are close, the curves are similar and although results differ from experiment to experiment, overall NLLS results give the best fit.

The results are summarized in Table 4 . As can be seen from the table, EKT and NLLS results are nearly equal with KT results slightly higher (closer to one). This is because the composition drift is not taken into account by the KT method.

The reactivity ratios found by the NLLS method are $r_{a}=$ $0.74, r_{b}=0.49$ and their product is $r_{a} \cdot r_{b}=0.36$. The reactivity ratios published by Roy et. al. [29] are much closer to one than our reactivity ratios presented in this paper (Table 4), so that the copolymer in the referred work is in almost perfectly random structure, while here the product of the reactivity ratios is 0.36 and there is a tendency to alternate.

3.2. Glass Transition Temperature. Differential scanning calorimetry (DSC) curves of all the copolymers are shown in Figure 6. All of copolymers are observed to have a single $T_{g}$ indicating the absence of a mixture of homopolymers or the formation of a block copolymer. The $T_{g}$ of poly(APMA) is at $146^{\circ} \mathrm{C}$ and that of poly(EMA) is at $84^{\circ} \mathrm{C}$. $T_{g}$ of copolymer is increased as the APMA fraction in the copolymer is increased.

3.3. Thermogravimetric Analysis (TGA). TGA thermograms of the polymers were shown in Figure 7. The thermograms 
TABLE 5: Thermal behaviour and decomposition temperatures of the copolymers and homopolymers.

\begin{tabular}{|c|c|c|c|c|c|c|c|c|}
\hline \multirow{2}{*}{ Polymer } & \multirow{2}{*}{$T_{g}\left({ }^{\circ} \mathrm{C}\right)$} & \multirow{2}{*}{$T_{\max }{ }^{\mathrm{a}}\left({ }^{\circ} \mathrm{C}\right)$} & \multirow{2}{*}{$\mathrm{IDT}^{\mathrm{b}}\left({ }^{\circ} \mathrm{C}\right)$} & \multirow[b]{2}{*}{$10 \%$} & \multicolumn{4}{|c|}{ Temperatures $\left({ }^{\circ} \mathrm{C}\right)$ at selected $\%$ weight loss } \\
\hline & & & & & $30 \%$ & $50 \%$ & $70 \%$ & $90 \%$ \\
\hline P(APMA) & 146 & 398 & 250 & 296 & 353 & 383 & 400 & 421 \\
\hline P(APMA0.88) & 138 & 275 & 256 & 271 & 309 & 370 & 396 & 422 \\
\hline P(APMA0.71) & 132 & 408 & 253 & 270 & 327 & 383 & 405 & 428 \\
\hline P(APMA0.56) & 125 & 404 & 253 & 272 & 350 & 387 & 407 & 436 \\
\hline P(APMA0.38) & 120 & 404 & 243 & 270 & 359 & 390 & 409 & 443 \\
\hline P(EMA) & 84 & 392 & 175 & 286 & 345 & 376 & 397 & 429 \\
\hline
\end{tabular}

${ }^{\mathrm{a}}$ Temperature for maximum rate of decomposition. ${ }^{\mathrm{b}}$ Initial decomposition temperature.

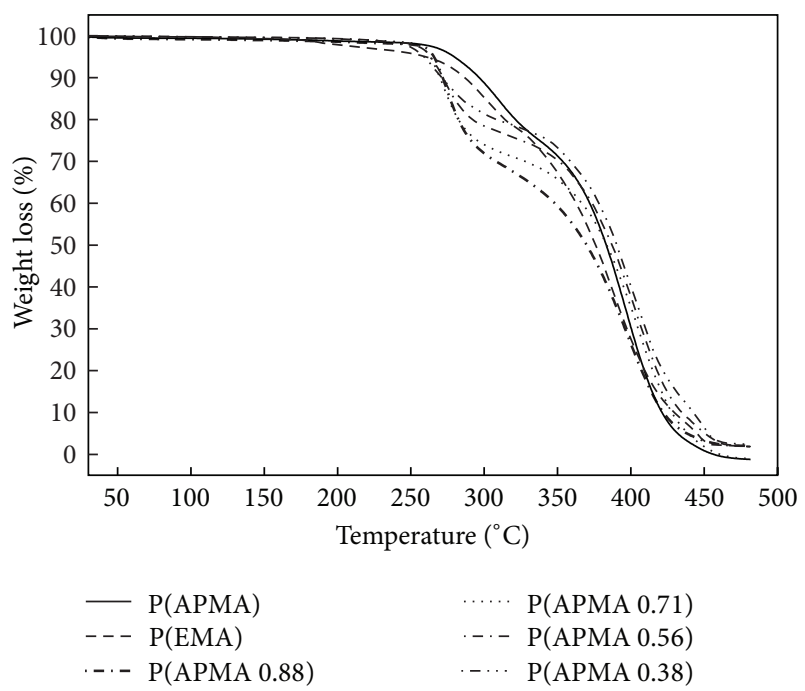

FIGURE 7: Thermal degradation curves of homo- and copolymers.

clearly indicate that all the polymers undergo two stages decomposition. The thermal behavior of any polymer should be affected appreciably by the introduction of a comonomer in the polymer chain. Thus it was important to investigate the thermal behavior of the prepared copolymers. $T_{g}$ 's, temperatures for maximum rate of decomposition, initial decomposition temperatures, and temperatures at selected \% weight loss are given in Table 5 .

For the study of the kinetics of the thermal degradation of polymers, there can be selected isothermal thermogravimetry (ITG) or thermogravimetry (TG) at different heating rates [37]. Although ITG is superior in obtaining an accurate activation energy for thermal degradation in cases where depolymerization is competing with cyclization or crosslinking due to the side groups, TG at different heating rates is much more convenient than ITG for the investigation of thermal degradation kinetics. Therefore, in this study, TG curves at various heating rates have been obtained and the activation energies $\left(\Delta E_{d}\right)$ for the thermal degradation of the polymers were calculated with Ozawa plots. Degradations have been performed in the scanning mode, from 35 to $500^{\circ} \mathrm{C}$, under a nitrogen flow $\left(20 \mathrm{~mL} \mathrm{~min}^{-1}\right)$, at various heating rates $\left(5.0,10.0,15.0\right.$, and $\left.25.0^{\circ} \mathrm{C} \mathrm{min}^{-1}\right)$. The TGA thermograms of poly(APMA) are given in Figure 8.

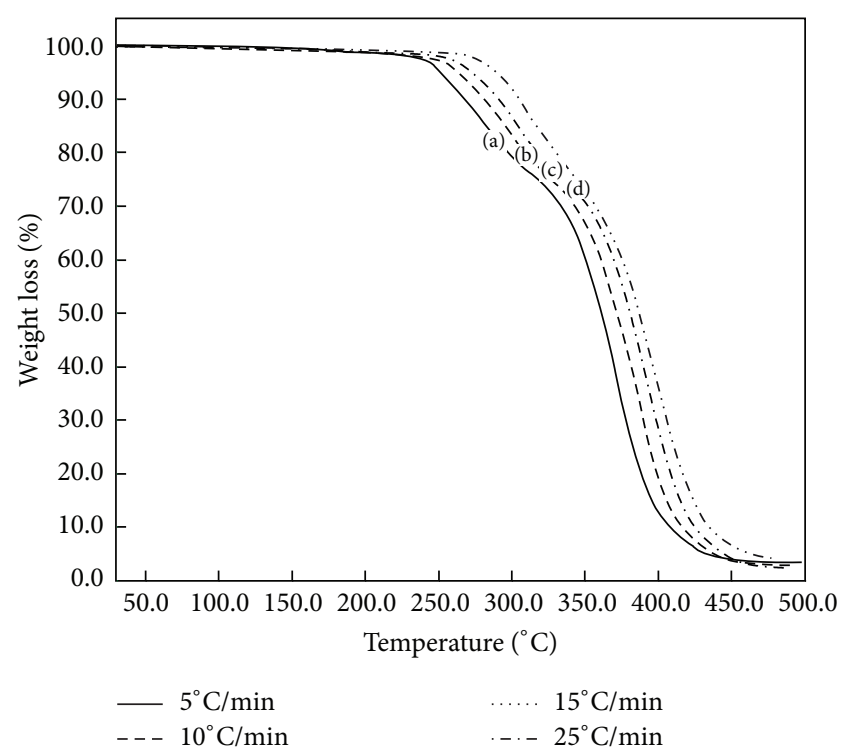

FIGURE 8: The thermal degradation curves of poly(APMA) at different heating rates.

According to the method of Ozawa [38], the apparent thermal decomposition activation energy, $E_{d}$, can be determined from the TGA thermograms under various heating rates as in the following equation:

$$
E_{d}=-\frac{R}{b}\left[\frac{d \log \beta}{d(1 / T)}\right] .
$$

Here $R$ has been the gas constant $(8.314 \mathrm{~J} / \mathrm{mol}), b$ is a constant $(0.4567)$, and $\beta$ has been the heating rate $\left({ }^{\circ} \mathrm{C} / \mathrm{min}\right)$. According to $(8)$, the activation energy of degradation can be determined from the slope of the linear relationship between $\log \beta$ and the $1 / T$. As shown in Figure 9, the activation energy has been found as $186 \mathrm{~kJ} / \mathrm{mol}$.

\section{Conclusions}

Copolymers of APMA with EMA were synthesized by free radical polymerization. The monomer reactivity ratios of the copolymers were calculated using KT, EKT, and linear and a nonlinear NLLS method. The results show that both monomer reactivity ratios are less than one, with APMA 


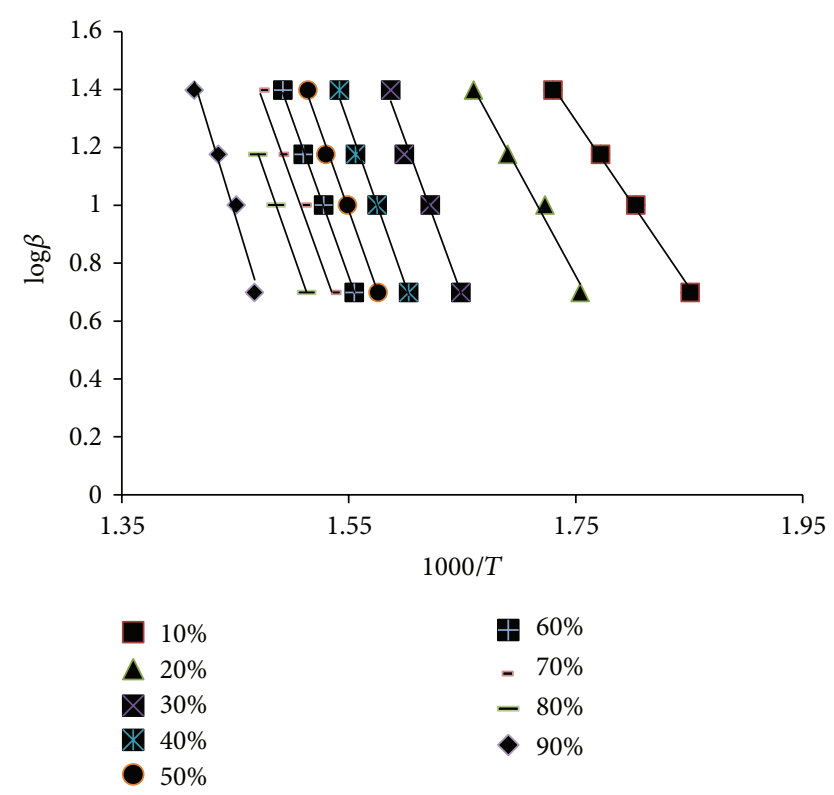

Figure 9: Ozawa plots of the logarithm of the heating rate $(\beta)$ versus the reciprocal temperature $(1000 / T)$ at different conversions for poly(APMA).

the more active monomer. All three methods gave consistent results. This leads to copolymers with a tendency for alternation and a slight composition drift.

Both molecular weights increased with increasing percentage of APMA in the feed composition. GPC data imply that the polydispersity index of the copolymers is between 1.25 and 1.4 and this implies a strong tendency for chain termination by coupling.

The DSC analysis indicated that the $T_{g}$ of copolymers increased with increase in APMA content. The thermal stability of the copolymers increased with the increase of APMA units in the copolymer. Initial decomposition temperature of all copolymers is higher than that of poly(EMA) (Table 5). Decomposition energy $\left(E_{d}\right)$ of poly(APMA) was found as $186 \mathrm{~kJ} / \mathrm{mol}$.

Poly-EMA is a soft methacrylate polymer and polyAPMA is a hard and strong one. Using the copolymer composition and thermal analysis results together it is possible to obtain copolymers with desired thermal properties.

\section{Conflict of Interests}

The authors declare that there is no conflict of interests regarding the publication of this paper.

\section{Acknowledgment}

Dr. Gamze Barim acknowledges the financial support provided by the Adiyaman University Research Fund (Project no. FEFBAP 2009-16).

\section{References}

[1] I. Erol and S. Kolu, "Copolymers of a new methacrylate monomer bearing oxime ester and ether with methyl methacrylate: synthesis, characterization, monomer reactivity ratios, and biological activity," Journal of Applied Polymer Science, vol. 120, no. 1, pp. 279-290, 2011.

[2] C. Soykan, A. Delibas, and R. Coskun, "Novel copolymers of 4chloronaphthyl methacrylate with acrylonitrile: determination of monomer reactivity ratios and antimicrobial activity," Journal of Macromolecular Science, Part A, vol. 46, no. 3, pp. 250-267, 2009.

[3] P. S. Vijayanand, C. S. Unnithan, A. Penlidis, and S. Nanjundan, "Copolymerization of benzoylphenyl methacrylate with methyl methacrylate: synthesis, characterization and determination of monomer reactivity ratios," Journal of Macromolecular Science, Part A, vol. 42, no. 5, pp. 555-569, 2005.

[4] T. Narasimhaswamy, S. C. Sumathi, and B. S. R. Reddy, "4acetylphenyl acrylate-glycidyl methacrylate copolymers: synthesis, characterization and reactivity ratios," European Polymer Journal, vol. 27, no. 3, pp. 255-261, 1991.

[5] K. Demirelli, E. Kaya, and M. Coşkun, "Polymers based on phenyl methacrylate: synthesis via atom transfer radical polymerization, characterization, monomer reactivity ratios, and thermal stabilities," Journal of Applied Polymer Science, vol. 99, no. 6, pp. 3344-3354, 2006.

[6] O. B. Abdyev, "Radical copolymerization of glycidyl methacrylate and carbonyl-containing phenyl methacrylates," Polymer Science Series B, vol. 53, no. 5-6, pp. 278-282, 2011.

[7] G. Kumar, N. Nisha, S. Mageswari, and K. Subramanian, "Synthesis, characterization of poly (4-benzyloxyphenylmethacrylate) and its copolymer with butyl methacrylate and determination of monomer reactivity ratios," Journal of Polymer Research, vol. 18, no. 2, pp. 241-250, 2011.

[8] S. K. Das and S. Lenka, "Polymers from renewable resources. XXIX. Synthesis and characterization of interpenetrating networks derived from castor-oil-based polyurethane-4-acetyl phenyl methacrylate," Polymer-Plastics Technology and Engineering, vol. 38, no. 1, pp. 149-157, 1999.

[9] U. Senthilkumar, R. Balaji, and S. Nanjundan, "Copolymerization of 2-(n-phthalimido)ethyl methacrylate with glycidyl methacrylate: synthesis, characterization, and monomer reactivity ratios," Journal of Applied Polymer Science, vol. 81, no. 1, pp. 96-103, 2001.

[10] L. J. F. Mary and A. V. R. Reddy, "Synthesis, characterization and thermal properties of copolymers of 3-hydroxy-4-acetylphenyl methacrylate with methyl acrylate," European Polymer Journal, vol. 27, no. 7, pp. 627-631, 1991.

[11] S. Mageswari and K. Subramanian, "Synthesis, characterization, and study of antibacterial activity of homopolymers and copolymers of 4-benzyloxyphenylacrylates for pressuresensitive adhesive application," Journal of Applied Polymer Science, vol. 125, no. 4, pp. 3115-3124, 2012.

[12] S. Nanjundan, C. S. Unnithan, C. S. J. Selvamalar, and A. Penlidis, "Homopolymer of 4-benzoylphenyl methacrylate and its copolymers with glycidyl methacrylate: synthesis, characterization, monomer reactivity ratios and application as adhesives," Reactive and Functional Polymers, vol. 62, no. 1, pp. 11-24, 2005.

[13] P. Samatha, T. Thimma Reddy, P. V. S. S. Srinivas, and N. Krishnamurti, "Effect of addition of various acrylates on the performance of ethyl cyanoacrylate adhesive," Polymer-Plastics Technology and Engineering, vol. 39, no. 2, pp. 381-392, 2000. 
[14] C. S. Jone Selvamalar, P. S. Vijayanand, A. Penlidis, and S. Nanjundan, "Homopolymer and copolymers of 4-benzyloxycarbonylphenyl acrylate with glycidyl methacrylate: synthesis, characterization, reactivity ratios, and application as adhesive for leather," Journal of Applied Polymer Science, vol. 91, no. 6, pp. 3604-3612, 2004.

[15] N. Soto, M. Sanmiguel, and F. Vázquez, "Synthesis and characterization of water-borne adhesives based on 2-ethylhexylacrylate-butylacrylate copolyrners functionalized with acrylic acid," International Journal of Polymeric Materials, vol. 54, no. 9, pp. 871-881, 2005.

[16] P. S. Vijayanand, C. S. J. Selvamalar, A. Penlidis, and S. Nanjundan, "Copolymers of 3,5-dimethylphenyl acrylate and methyl methacrylate: synthesis, characterization and determination of monomer reactivity ratios," Polymer International, vol. 52, no. 12, pp. 1856-1862, 2003.

[17] P. G. Vijayaraghavan and B. S. R. Reddy, "4-Chlorophenyl acrylate and glycidyl methacrylate copolymers: synthesis, characterization, reactivity ratios, and application," Journal of Macromolecular Science, Part A, vol. 36, no. 9, pp. 1181-1195, 1999.

[18] J. L. Hua, J. W. Y. Lam, H. Dong, L. Wu, K. S. Wong, and B. Z. Tang, "Synthesis, light emission, and photo-cross-linking of luminescent polyacetylenes containing acrylic pendant groups," Polymer, vol. 47, no. 1, pp. 18-22, 2006.

[19] K. Ichimura and Y. Nishio, "Photocrosslinkable polymers having p-phenylenediacrylate group in the side chain: argon laser photoresist," Journal of Polymer Science, Part A, vol. 25, no. 6, pp. 1579-1590, 1987.

[20] R. Balaji, D. Grande, and S. Nanjundan, "Photoresponsive polymers having pendant chlorocinnamoyl moieties: synthesis, reactivity ratios and photochemical properties," Polymer, vol. 45, no. 4, pp. 1089-1099, 2004.

[21] A. Arun and B. S. R. Reddy, "In vitro drug release studies from the polymeric hydrogels based on HEA and HPMA using 4\{(E)-[(3Z)-3-(4-(acryloyloxy)benzylidene)-2-hexylidene]methyl\} phenyl acrylate as a crosslinker," Biomaterials, vol. 26, no. 10, pp. 1185-1193, 2005.

[22] S. C. Pandeya, N. Rathor, and A. Singh, "Synthesis and characterisation of biologically active barium containing polymer films," Journal of Polymer Materials, vol. 16, no. 3, pp. 253-258, 1999.

[23] M. Jöhnck, L. Müller, A. Neyer, and J. W. Hofstraat, "Copolymers of halogenated acrylates and methacrylates for the application in optical telecommunication: optical properties, thermal analysis and determination of unsaturation by quantitative FT-Raman and FT-IR spectroscopy," European Polymer Journal, vol. 36, no. 6, pp. 1251-1264, 2000.

[24] R. V. Yarapathi, S. Kurva, and S. Tammishetti, "Synthesis of 3,4-dihydropyrimidin-2(1H)ones using reusable poly(4vinylpyridine-co-divinylbenzene)-Cu(II)complex," Catalysis Communications, vol. 5, no. 9, pp. 511-513, 2004.

[25] N. Ayaz, F. Bezgin, and K. Demirell,, "Polymers based on methacrylate bearing coumarin group: synthesis via free radical polymerization, monomer reactivity ratios, dielectric behavior, and thermal stabilities," ISRN Polymer Science, vol. 2012, Article ID 352759, 13 pages, 2012.

[26] P. S. Vijayanand, S. Kato, S. Satokawa, M. Kishimoto, and T. Kojima, "Synthesis, characterization and thermal properties of homo and copolymers of 3,5-dimethoxyphenyl methacrylate with glycidyl methacrylate: determination of monomer reactivity ratios," Reactive and Functional Polymers, vol. 69, no. 6, pp. 333-340, 2009.
[27] G. Barim, K. Demirelli, and M. Coşkun, "Conventional and atom transfer radical copolymerization of phenoxycarbonylmethyl methacrylate-styrene and thermal behavior of their copolymers," Express Polymer Letters, vol. 1, no. 8, pp. 535-544, 2007.

[28] G. J. Reddy, S. V. Naidu, and A. V. Rami Reddy, "Synthesis and Characterization of Poly(N-phenyl methacrylamide-co-methyl methacrylate) and Reactivity Ratios Determination," Journal of Applied Polymer Science, vol. 90, no. 8, pp. 2179-2186, 2003.

[29] S. G. Roy, K. Bauri, S. Pal, A. Goswami, G. Madras, and P. De, "Synthesis, characterization and thermal degradation studies of dual temperature- and $\mathrm{pH}$-sensitive RAFT-made copolymers of $\mathrm{N}, \mathrm{N}$-(dimethylamino)ethyl methacrylate and methyl methacrylate," Polymer International, vol. 62, no. 3, pp. 463-473, 2013.

[30] S. Thamizharasi, P. Gnanasundaram, and B. S. R. Reddy, "4acetylphenyl methacrylate-co-glycidyl methacrylate polymers: synthesis, characterization and reactivity ratios," Journal of Polymer Materials, vol. 15, no. 3, pp. 229-236, 1998.

[31] T. Kelen and F. Tüdös, "Analysis of linear methods for determining copolymerization reactivity ratios, I. New improved linear graphic method," Journal of Macromolecular Science. Chemistry A, vol. 9, no. 1, pp. 1-27, 1975.

[32] T. Kelen, F. Tudos, B. Turcsanyi, and J. P. Kennedy, "Analysis of the linear methods for determining copolymerization reactivity ratios. IV. A comprehensive and critical reexamination of carbocationic copolymerization data," Journal of Polymer Science: Polymer Chemistry Edition, vol. 15, no. 12, pp. 3047-3074, 1977.

[33] D. Sünbül, H. Çatalgil-Giz, W. Reed, and A. Giz, "An error-invariables method for determining reactivity ratios by on-line monitoring of copolymerization reactions," Macromolecular Theory and Simulations, vol. 13, no. 2, pp. 162-168, 2004.

[34] Z. M. O. Rzaev, A. Güner, G. Kibare, H. Kaplan Can, and A. Așc, "Terpolymerization of maleic anhydride, trans-stilbene and acrylic monomers," European Polymer Journal, vol. 38, no. 6, pp. 1245-1254, 2002.

[35] S. Teramachi, A. Hasegawa, M. Akatsuka, A. Yamashita, and N. Takemoto, "Molecular Weight Distribution and correlation between chemical composition and Molecular Weight in a high-conversion copolymer of styrene-methyl acrylate," Macromolecules, vol. 11, no. 6, pp. 1206-1210, 1978.

[36] F. R. Mayo and F. M. Lewis, "Copolymerization. I. A basis for comparing the behavior of monomers in copolymerization; the copolymerization of styrene and methyl methacrylate," Journal of the American Chemical Society, vol. 66, no. 9, pp. 1594-1601, 1944.

[37] W. I. Kim, S. D. Kim, S. B. Lee, and I. K. Hong, "Kinetic characterization of thermal degradation process for commercial rubbers," Journal of Industrial and Engineering Chemistry, vol. 6, no. 5, pp. 348-355, 2000.

[38] J. H. Flynn and L. A. Wall, "A quick, direct method for the determination of activation energy from thermogravimetric data," Journal of Polymer Science, Polymer Letters, vol. 4, no. 5, pp. 323-328, 1966. 

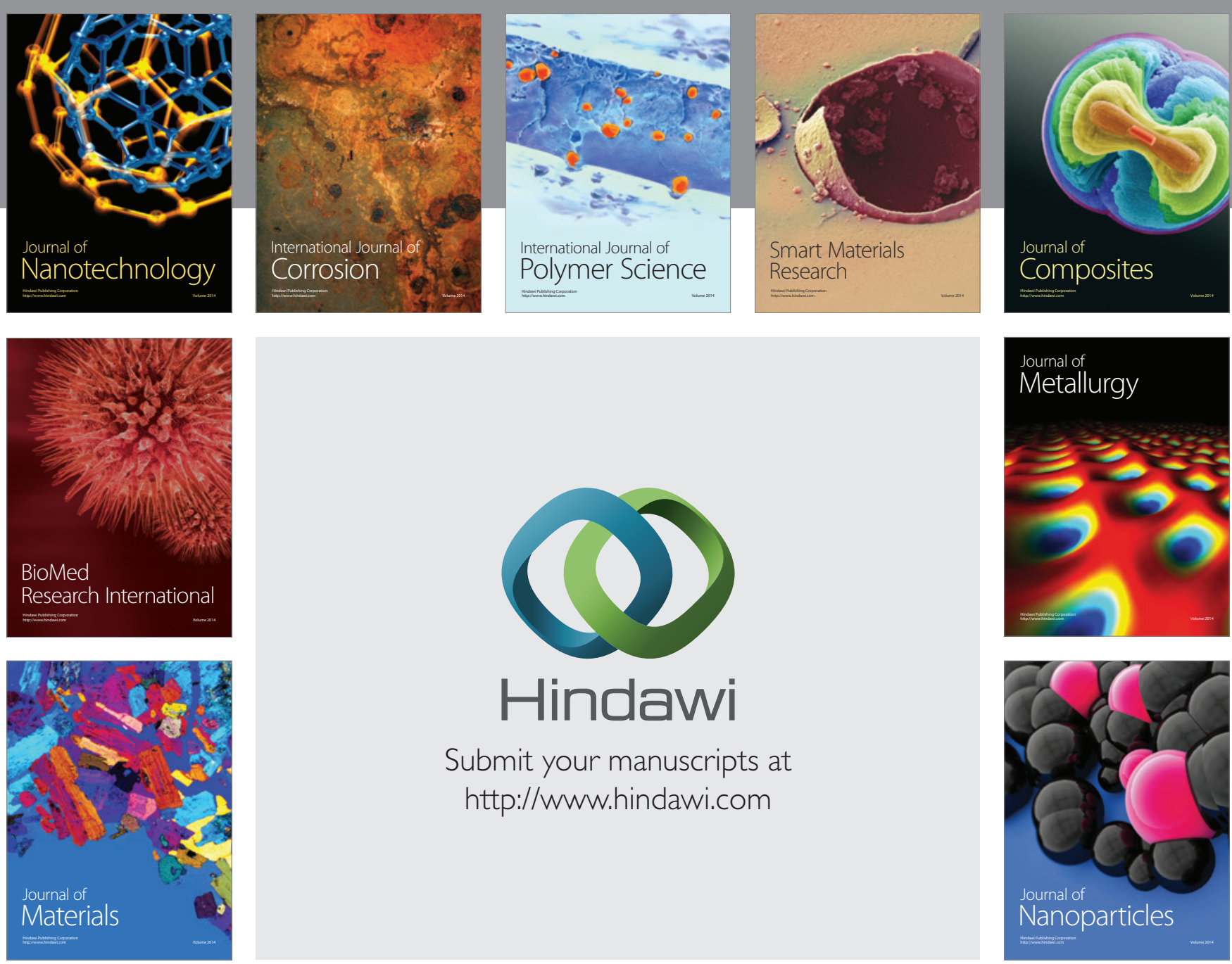

Submit your manuscripts at http://www.hindawi.com
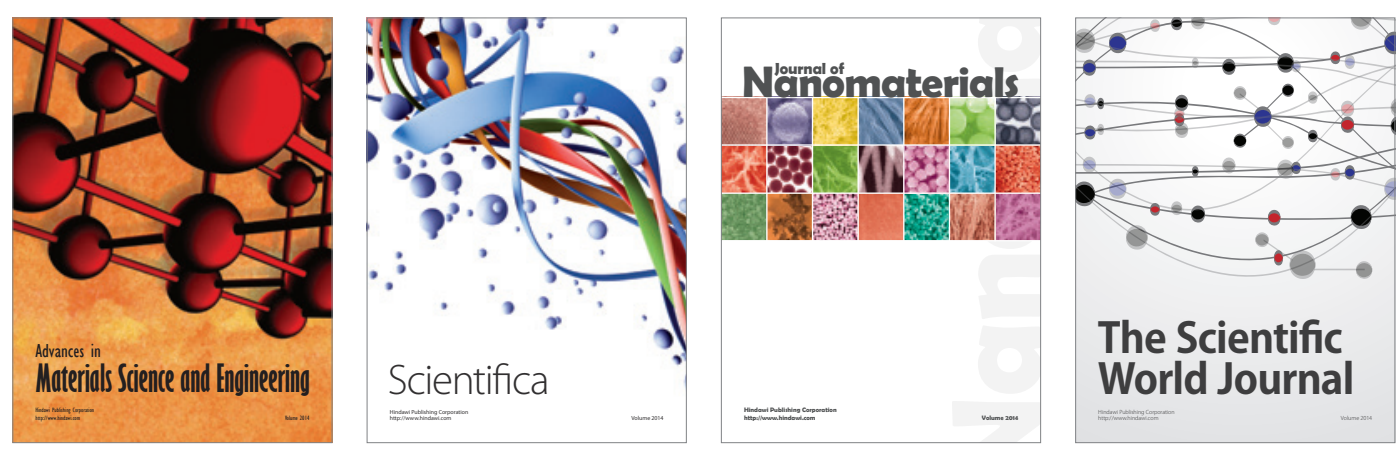

\section{The Scientific World Journal}
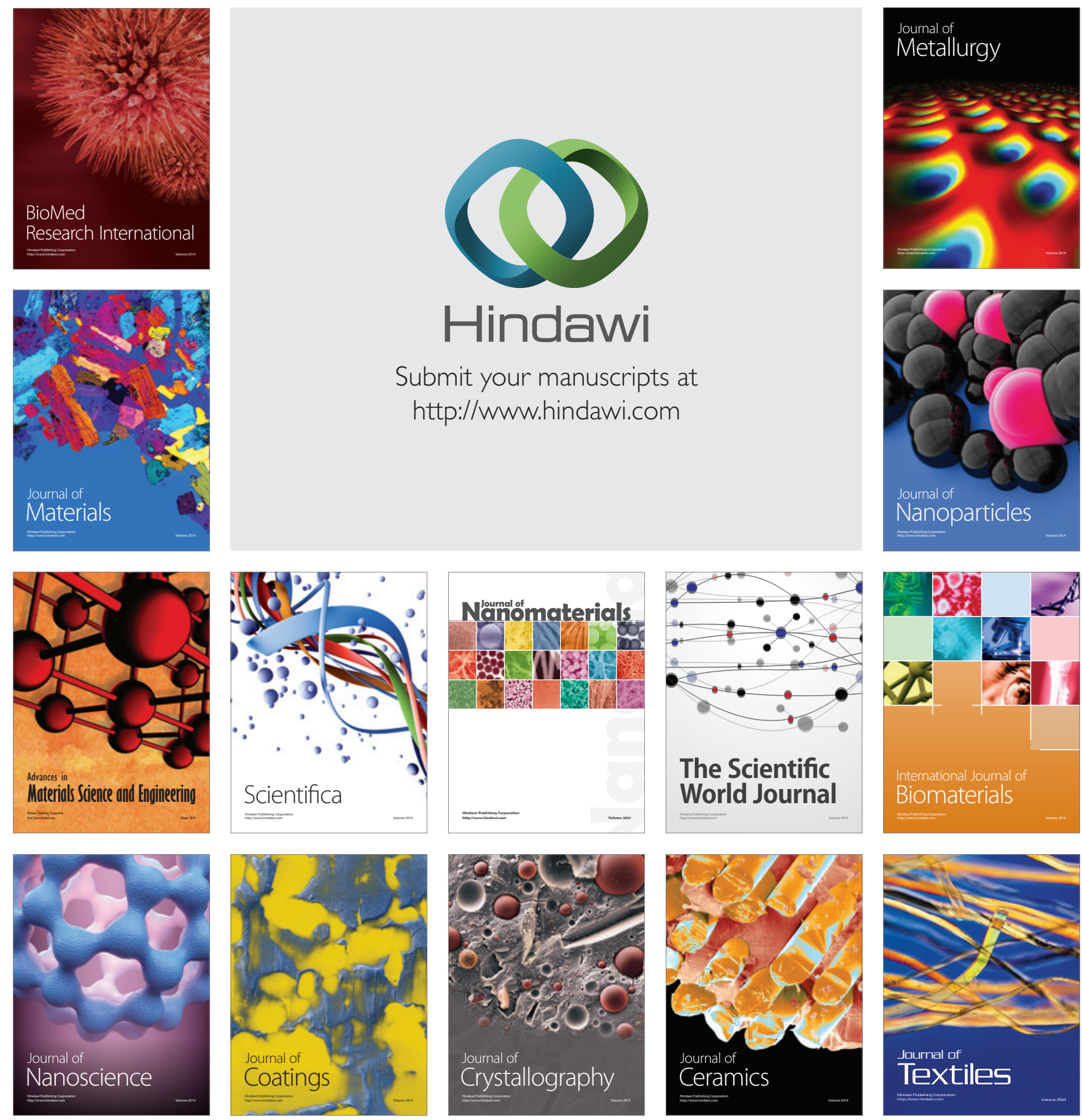